'Sección de Reumatología,

Departamento de Medicina Interna, Universidad de Concepción. Concepción, Chile. Servicio de Reumatología, Hospital Clínico Regional de Concepción. Concepción, Chile. 2Departamento de Patología, Hospital Clínico Regional de Concepción. Concepción, Chile. anterna, Facultad de Medicina, Universidad de Concepción. Concepción, Chile.

No hubo fuente de apoyo financiero. Autores declaran no tener conflictos de interés.

Recibido el 26 de marzo de 2017, aceptado el 29 de mayo de 2018 .

Correspondencia a: Consuelo Patricia Arroyo Schumacher Cirujano Guzmán \#70. Providencia. Región Metropolitana. caschuma91@gmail.com

\section{Mieloma múltiple en pacientes con lupus eritematoso sistémico: presentación de dos casos}

\author{
IRENE CASTRO ${ }^{1}$, CONSUELO ARROYO S. ${ }^{\text {a }}$, \\ VALENTINA YÁÑEZ, CLAUDIA CABEZAS ${ }^{2}$
}

\section{Multiple myeloma in systemic lupus erythematosus. Report of two cases}

There is an increased incidence of hematological malignancies, particularly non-Hodgkin lymphoma (NHL) in systemic lupus erythematosus (SLE). In contrast, the concurrence with Multiple Myeloma (MM) is very rare, and the possible pathogenetic mechanisms underlying this association remain unclear. We report two patients who developed MM 15 and four years after being diagnosed as having SLE.

(Rev Med Chile 2018; 146: 670-674)

Key words: Hematologic Neoplasms; Lupus Erythematosus, Systemic; Multiple Myeloma.

\section{E} 1 Lupus Eritematoso Sistemico (LES) es de las enfermedades autoinmunes sistémicas más frecuentes ${ }^{1}$. Mientras la mortalidad atribuida a sus manifestaciones y tratamiento ha disminuido, los pacientes aun fallecen de enfermedad cardiovascular, infecciones y cáncer $^{2}$. Se ha establecido una asociación con riesgo aumentado de cáncer, principalmente neoplasias hematológicas. Particularmente se ha observado aumento de incidencia y mortalidad de Linfoma no Hodgkin (LNH), comparado con las tasas esperadas de cáncer en población general ${ }^{3,4}$. Un estudio sugiere que el riesgo de desarrollar Mieloma múltiple (MM) también puede estar aumentado en pacientes con LES ${ }^{5}$. Está concurrencia es rara y ha habido pocos reportes de casos en la literatura. Choi et al. en marzo de 2010 reportó 13 casos clínicos, incluyendo el suyo, hacia esa fecha ${ }^{6}$.

Se presentarán dos casos clínicos de esta asociación; la incidencia de neoplasias hematológicas, específicamente el MM de la línea celular linfoide, en pacientes con LES.

\section{Caso 1}

Paciente masculino de 49 años con antecedentes de Hipotiroidismo, Hipertensión arterial, Cardiopatía coronaria isquémica y LES diagnosticado en 1997 a los 31 años (artritis no erosiva muñecas, codo izquierdo, rodillas, fotosensibilidad, eritema facial, anemia hemolítica, leucopenia, AAN + y aDNA + , hipocomplementemia), tratado con corticoides y posteriormente se asocia azatioprina.

En octubre de 2012 consultó por dolor en muslo derecho. Resonancia Nuclear Magnetica (RNM) de columna evidenció lesiones metastásicas de T12-L2-L4-L5, sacro e íleon. Se hospitalizó para estudio y manejo de Hipercalcemia maligna. TAC de cuello reveló lesiones osteolíticas en ala mayor y menor de esfenoides y en seno esfenoidal derecho de aspecto infiltrativo. Cintigrama óseo mostró actividad osteoblástica anormal en cráneo, esternón, columna vertebral, arcos costales, y pelvis sugerentes de atipía ósea secundaria. Biopsia esfenoidal informó fragmentos de mucosa nasal infiltradas por células plasmáticas monoclona- 
les compatibles con Plasmacitoma (Figura 1). Electroforesis de proteínas en sangre con banda monoclonal en beta $35,4 \%$; región gamma disminuida. Cuantificación Inmunoglobulina G (IgG) $3.930 \mathrm{mg} / \mathrm{dl}$ (normal $680-1.450 \mathrm{mg} / \mathrm{dL}$ ), Inmunoglobulina A (IgA) $140 \mathrm{mg} / \mathrm{dl}$ (normal 83-407 mg/ dL), Inmunoglobulina E (IgE) 18,3 UI/dl (normal $<100 \mathrm{mg} / \mathrm{dL}$ ), Inmunoglobulina M (IgM) 23,2 $\mathrm{mg} / \mathrm{dl}$ (normal 74-278 mg/dL), Albúmina 2,5 g/ dL, Proteínas totales $8,3 \mathrm{~g} / \mathrm{dL}$, Hemoglobina 10,7 $\mathrm{g} / \mathrm{dL}$, recuento de glóbulos blancos $6.600 \times 10^{3} / \mathrm{uL}$, linfocitos $800 \times 10^{3} / \mathrm{uL}$, plaquetas $236.000 \times 10^{3 /}$ uL. Inmunofijación positiva para cadenas lambda. Beta 2 Microglobulina 2,81 mg/dl. Mielograma mostró células plasmáticas $<10 \%$ y compromiso parcheado de ésta. Radiografía de cráneo con lesiones líticas.

Se diagnosticó a los 46 años MM sintomático IgG cadenas livianas lambda, según criterios del International Myeloma Working Group. Etapa III A según etapificación de Durie y Salmon y etapa
II según el Sistema de Etapificación Internacional (ISS) $)^{7,8}$.

Inició tratamiento con Ciclofosfamida $50 \mathrm{mg}$ día, Talidomida $100 \mathrm{mg}$ día por medio, Dexametasona $40 \mathrm{mg}$ día y Pamidronato $90 \mathrm{mg}$ mensual. Por compromiso de $40 \%$ cuerpo y muro posterior vertebral y riesgo de fractura de cadera se indicó radioterapia en pelvis. Respuesta completa tras 12 meses de tratamiento. Se programó trasplante autólogo de médula ósea (TAMO), no realizado por obtención insuficiente de progenitores hematopoyéticos y complicaciones infecciosas secundarias.

En diciembre de 2014, por pesquisa en Cintigrama óseo de actividad osteoblástica aumentada multifocal, IgG en aumento y Electroforesis de proteínas con componente monoclonal se indicó quimioterapia de segunda línea con Vincristina, Doxorrubicina y Dexametasona (VAD), completó 5 ciclos. Control de inmunoglobulinas mostró IgA $169 \mathrm{mg} / \mathrm{dL}$, IgG $7.010 \mathrm{mg} / \mathrm{dL}$ e IgM 16,8 mg/dL

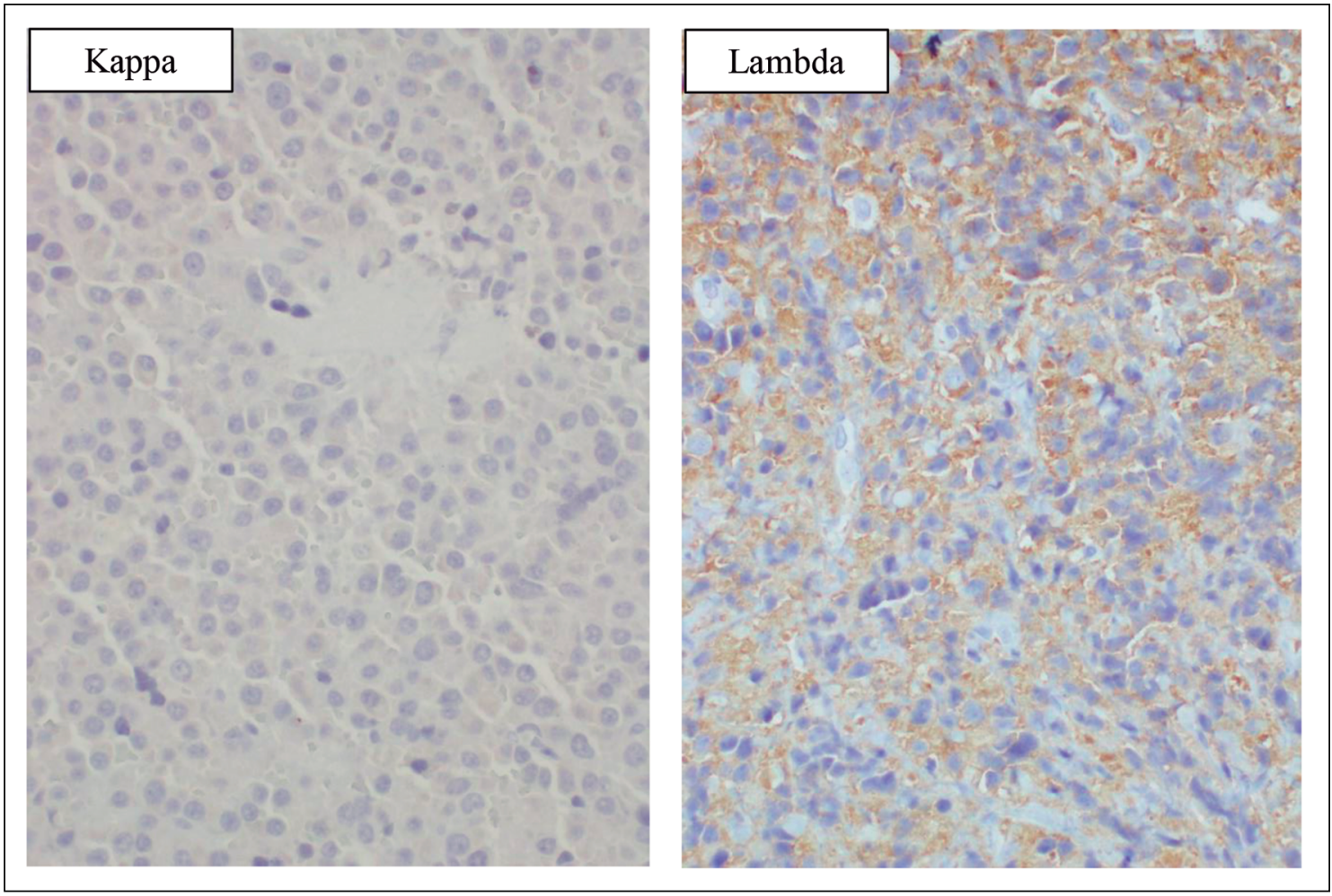

Figura 1. Biopsia de hueso esfenoidal: Inmunohistoquímica demuestra monoclonalidad de células plasmáticas (CD138+), con restricción de cadenas livianas (Lambda +/ Kappa -) y un índice de proliferación Ki-67 de 40\%. LCA, CD3 CD20 y Pax-5 fueron negativos (400x). 
con componente monoclonal beta de $37,8 \%$. En mayo de 2016 consultó por aumento de volumen cervical. TAC de cuello y tórax evidenció masa retrofaríngea con compromiso vertebral de C2, canal raquídeo y adenopatías cervicales. No se realizó biopsia, ya que evolucionó con hipercalcemia maligna, aumento de la actividad lúpica y anemia severa con mala respuesta a tratamiento médico y radioterapia. Falleció el 02 de junio de 2016.

\section{Caso 2}

Paciente femenina de 54 años, con antecedentes de Hipertensión arterial y LES diagnosticado en 2006 a los 45 años con biopsia renal que evidenció nefropatía lúpica tipo IV, ANA positivo 1/160 granular y aDNA positivo. Recibió inducción con ciclofosfamida, mantención con prednisona y azatioprina, permaneciendo sedimento de orina inactivo y función renal normal.

En junio de 2010 destacó Velocidad de eritrosedimentación (VHS) $112 \mathrm{~mm} / \mathrm{h}$, aDNA negativo, ANA positivo 1/640, C3 $120 \mathrm{mg} / \mathrm{dL}, \mathrm{C} 416 \mathrm{mg} /$ dL, Hematocrito 36,3\%, Hemoglobina 12,7 g/ $\mathrm{dL}$, glóbulos blancos $8.500 \times 10^{3} / \mathrm{uL}$ con recuento diferencial normal, plaquetas $308.000 \times 10^{3} / \mathrm{uL}$, Creatinina $1,32 \mathrm{mg} / \mathrm{dL}$, orina completa normal. Sin signos clínicos de actividad lúpica. Por VHS persistentemente alta, se solicitó estudio de MM. Cuantificación de IgA $4.110 \mathrm{mg} / \mathrm{dL}$ (normal 83$407 \mathrm{mg} / \mathrm{dL}$ ), IgG $414 \mathrm{mg} / \mathrm{dL}$ (normal 680-1.450 $\mathrm{mg} / \mathrm{dL}$ ), IgM $48 \mathrm{mg} / \mathrm{dL}$ (normal 74- $278 \mathrm{mg} /$ $\mathrm{dL}$ ). Electroforesis de proteínas en sangre con componente monoclonal en Alfa 2 y Beta (Figura 2), Albúmina 3,5 g/dL. Beta 2 Microglobulina 2,9 $\mathrm{mg} / \mathrm{dL}$. Radiografías de huesos largos normal. Con inmunofijación en suero positivo para IgA kappa. En mayo de 2011 Mielograma mostró 47\% de células plasmáticas y mielomatosis medular.

Se diagnosticó a los 49 años MM asintomático IgA, según los criterios del International Myeloma Working Group. Etapa I según etapificación de Durie y Salmon y etapa I según ISS 7,8 .

Tras dos semanas del diagnóstico, presentó fractura vertebral. Biopsia de fragmentos vertebrales torácicos informó infiltración por Plasmocitoma Plasmocítico. Con esto se reclasificó diagnóstico como MM sintomático etapa III A, según Durie y Salmon. Se inició tratamiento con Pamidronato $90 \mathrm{mg}$ mensual, Talidomida $50 \mathrm{mg} /$ día por 10 días y Dexametasona $40 \mathrm{mg} /$ día días 1 -4 y 9-12, cada 2 meses completando 12 ciclos.

En junio de 2011, por fractura de T8, se realiza fijación de columna dorsal de T6 a T10. En agosto de 2011 inicia radioterapia paliativa sobre columna dorsal y sacroilíaca izquierda. Por mala tolerancia, cambió esquema a Melfalán $6 \mathrm{mg}$ y Prednisona $60 \mathrm{mg}$ por 7 días, cada 3 meses completando 8 ciclos y 2 años con Pamidronato. Al año de tratamiento en remisión completa. Control de inmunoglobulinas con IgA $425 \mathrm{mg} / \mathrm{dL}$, IgG $1.180 \mathrm{mg} / \mathrm{dL}$ e IgM 67mg/dL. Paciente rechazó TAMO y permanece en seguimiento.

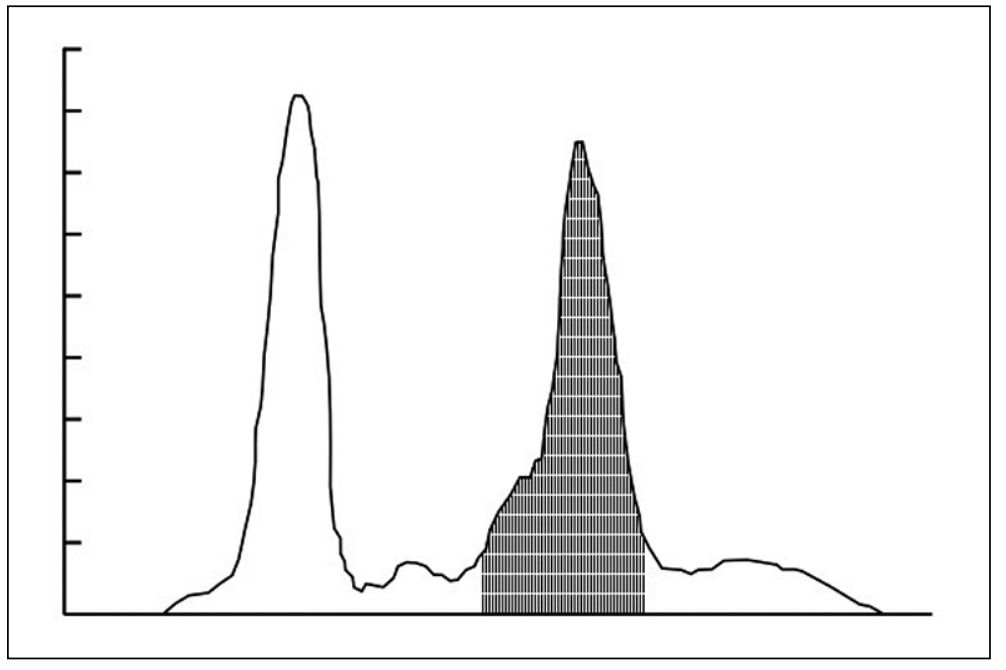

Figura 2. Electroforesis de proteínas en sangre. Muestra componente monoclonal en fracción alfa 2 y beta. SMC (Spike monoclonal component) $33,2 \%(2,6 \mathrm{~g} /$ $\mathrm{dL}$ ). Albúmina 3,3 g/dL, Alpha $10,3 \mathrm{~g} /$ $\mathrm{dL}$, Alpha 2 y Beta $4,3 \mathrm{~g} / \mathrm{dL}$, Gama 0,8 $\mathrm{g} / \mathrm{dL}$ y Proteínas totales $8,8 \mathrm{~g} / \mathrm{dL}$ (Fecha: 11.11.2010). 


\section{Discusión}

Se presentan dos casos clínicos de pacientes con LES asociado a MM en su evolución. Uno de sexo masculino que desarrolla $\mathrm{MM}$ a los 46 años, y otro femenino con diagnóstico a los 49 años, 15 y 4 años posterior al diagnóstico de LES, respectivamente.

MM es una neoplasia caracterizada por proliferación clonal de células plasmáticas malignas en la médula ósea produciendo proteínas monoclonales en sangre $u$ orina ${ }^{9}$. La edad media al diagnóstico es 66 años, siendo sólo 10\% y 2\% de los pacientes menores de 50 y 40 años, respectivamente $^{10,11}$.

Hace más de 30 años se ha sugerido una incidencia aumentada de cánceres hematológicos en pacientes con LES ${ }^{12}$. Un metaanálisis de estudios de cohorte demostró que LES puede aumentar 3 veces la tasa de incidencia estandarizada para neoplasias hematológicas ${ }^{13}$.

Para todas las formas de cánceres hematológicos se ha demostrado un mayor riesgo en LES, pero éste es notable para $\mathrm{LNH}^{3,13}$, aumentando el riesgo 6 veces. En el grupo de las neoplasias hematológicas no linfoma, las más frecuentes son las de tipo mieloide ${ }^{13}$. En cambio, en la población general, las neoplasias de tipo linfoide son 1,7 veces más frecuentes que las de tipo mieloide, al excluir los linfomas ${ }^{14}$. A pesar de que estudios anteriores no han demostrado que los pacientes con LES tienen mayor riesgo de MM, un estudio demostró que los pacientes con historia familiar de LES tienen mayor riesgo de desarrollar $\mathrm{MM}^{15}$, y también habría mayor ocurrencia de LES en los parientes de primer grado de personas con $\mathrm{MM}^{16}$.

Apor et al. demostró en población lúpica un riesgo 3 veces mayor de Linfoma Hodgkin (LH) y 2 veces mayor de Leucemia, y sólo un posible mayor riesgo de desarrollar $\mathrm{MM}^{13}$. Lu et al. en su estudio de cohorte de LES obtuvo una edad promedio de diagnóstico de MM de 49 años (rango 45-57), y la duración promedio de LES al momento del diagnóstico de MM fue de 17 años (rango 8-25) ${ }^{17}$. Estos datos son consistentes con lo expuesto en este reporte. En la revisión de casos clínicos de Choi et al. la edad media de diagnóstico de MM fue de 54 años, alrededor de 10 años antes que en la población general. Esto sugiere que factores genéticos o inmunológicos involucrados en el LES, pueden afectar la evolución molecular hacia MM quizás a edades más tempranas que la población general ${ }^{6}$.

Los mecanismos patogénicos de esta asociación no han sido completamente dilucidados. Se postula que un aumento en la actividad lúpica podría mediar este riesgo aumentado de malignidad hematológica. Sin embargo, un estudio de cohorte internacional no logró demostrar una actividad aumentada de LES en los casos de linfoma ${ }^{18}$. Se han propuesto diversas teorías para explicar esta coexistencia, entre ellas: la hiperactividad de células $B$ que favorece el escape anormal de clones de los mecanismos de regulación, una vigilancia defectuosa de células malignas y el rol de los agentes inmunosupresores usados para el control de la actividad del lupus. En el modelo de lupus animal, más de 30\% de los ratones mostraron Gammapatía monoclonal con una mayor prevalencia de $\mathrm{MM}^{19}$.

En consecuencia, hay que estar atentos a las manifestaciones clínicas y/o de laboratorio que no son explicadas por actividad lúpica y se sugiere considerar patologías oncohematológicas, como el $\mathrm{MM}$, entre las posibilidades diagnósticas.

\section{Referencias}

1. Tsokos GC. Systemic Lupus Erythematosus. N Engl J Med 2011; 365: 2110-21.

2. Ippolito A, Petri M. An update on mortality in systemic lupus erythematosus. Clin Exp Rheumatol 2008; 26 (5 Suppl 51): S72-9.

3. Bernatsky S, Ramsey-Goldman R, Labrecque J, Joseph L, Boivin JF, Petri M, et al. Cancer risk in systemic lupus: an updated international multi-centre cohort study. J Autoimmun 2013; 42: 130-5.

4. Bernatsky S, Boivin JF, Joseph L, Manzi S, Ginzler E, Gladman DD, et al. Mortality in systemic lupus erythematosus. Arthritis Rheum 2006; 54 (8): 2550-7.

5. Ali YM, Urowitz MB, Ibáñez D, Gladman DD. Monoclonal gammopathy in systemic lupus erythematosus. Lupus 2007; 16 (6): 426-9.

6. Choi JW, Han SW, Kwon KT, Kim GW. Early onset multiple myeloma in a patient with systemic lupus erythematosus: a case report and literature review. Clin Rheumatol 2010; 29: 1323-6.

7. Greipp PR, San Miguel J, Durie BG, Crowley JJ, Barlogie $\mathrm{B}$, Bladé J, et al. International staging system for multiple myeloma. J Clin Oncol 2005; 23 (15): 3412-20. 
8. Durie BG, Salmon SE. A clinical staging system for multiple myeloma. Cancer 1975; 36 (3): 842-54.

9. Kyle RA, Rajkumar SV. Multiplemyeloma. N Engl J Med 2004; 351 (18): 1860-73. Erratum in N Engl J Med 2005; 352 (11): 1163.

10. Kyle RA, Gertz MA, Witzig TE, Lust JA, Lacy MQ, Dispenzieri A, et al. Review of 1027 patients with newly diagnosed multiple myeloma. Mayo Clin Proc 2003; 78 (1): 21-33.

11. Blade J, Kyle RA. Multiple myeloma in young patients: clinical presentation and treatment approach. Leuk Lymphoma 1998; 30 (5-6): 493-501.

12. Green JA, Dawson AA, Walker W. Systemic lupus erythematosus and lymphoma. Lancet 1978; 312 (8093): 753-6.

13. Apor E, O’Brien J, Stephen M, Castillo JJ. Systemic lupus erythematosus is associated with increased incidence of hematologic malignancies: a meta-analysis of prospective cohort studies. Leuk Res 2014; 38 (9): 1067-71.

14. Howlader N, Noone AM, Krapcho M, Neyman N, Aminou R, Waldron W, et al. SEER Cancer Statistics Review,
1975-2009 (Vintage 2009 Populations). Bethesda, National Cancer Institute, 2009.

15. Landgren O, Linet MS, McMaster ML, Gridley G, Hemminki K, Gold LR. Familial characteristics of autoimmune and hematologic disorders in 8,406 multiple myeloma patients: a population-based case-control study. Int J Cancer 2006; 118 (12): 3095-8.

16. Anderson LA, Gadalla S, Morton LM, Landgren O, Pfeiffer R, Warren JL, et al. Population-based study of autoimmune conditions and the risk of specific lymphoid malignancies. Int J Cancer 2009; 125 (2): 398-405.

17. Lu M, Bernatsky S, Ramsey-Goldman R, Petri M, Manzi S, Urowitz MB, et al. Non-lymphoma hematological malignancies in systemic lupus erythematosus. Oncology 2013; 85 (4): 235-40.

18. Bernatsky S, Ramsey-Goldman R, Joseph L, Boivin JF, Costenbader K, Urowitz M, et al. Lymphoma risk in systemic lupus: effects of disease activity versus treatment. Ann Rheum Dis 2014; 73 (1): 138-42.

19. Sugai S, Pillarisetty R, Talal N. Monoclonal macroglobulinemia in NZB-NZW F1 mice. J Exp Med 1973; 138 (4): 989-1002. 